\title{
Mitochondrial Fusion and Fission in Mammals
}

\author{
David C. Chan
}

Division of Biology, California Institute of Technology, Pasadena, California; email: dchan@caltech.edu

Annu. Rev. Cell Dev. Biol. 2006. 22:79-99

First published online as a Review in Advance on May 11, 2006

The Annual Review of

Cell and Developmental Biology is online at http://cellbio.annualreviews.org

This article's doi:

10.1146/annurev.cellbio.22.010305.104638

Copyright (c) 2006 by Annual Reviews. All rights reserved

$1081-0706 / 06 / 1110-0079 \$ 20.00$

\section{Key Words}

mitochondrial dynamics, organelle morphology, membrane fusion, membrane trafficking

\begin{abstract}
Eukaryotic cells maintain the overall shape of their mitochondria by balancing the opposing processes of mitochondrial fusion and fission. Unbalanced fission leads to mitochondrial fragmentation, and unbalanced fusion leads to mitochondrial elongation. Moreover, these processes control not only the shape but also the function of mitochondria. Mitochondrial dynamics allows mitochondria to interact with each other; without such dynamics, the mitochondrial population consists of autonomous organelles that have impaired function. Key components of the mitochondrial fusion and fission machinery have been identified, allowing initial dissection of their mechanisms of action. These components play important roles in mitochondrial function and development as well as programmed cell death. Disruption of the fusion machinery leads to neurodegenerative disease.
\end{abstract}




\section{Contents}

INTRODUCTION .............. 80

THE FUSION MACHINERY . . . . . 80

Mitofusins: Mfn1 and Mfn2 ..... 81

OPA1 ..................... 81

THE FISSION MACHINERY ..... 83

Dynamin-Related Protein 1..... 83

Fis $1 \ldots \ldots \ldots \ldots \ldots \ldots \ldots . . . \ldots 3$

OTHER PLAYERS ............. 83

MOLECULAR MECHANISM ..... 85

Fusion Mechanism ............. 85

Fission Mechanism ............. 87

BIOLOGICAL SIGNIFICANCE ... 88

Cell Biology of Fusion and Fission 88

Mitochondrial Dynamics in

Development and Apoptosis ... 89

Mitochondrial Dynamics in

Disease ................. 91

PERSPECTIVES .............. 92

\section{INTRODUCTION}

In the past several years, there have been tremendous advances in our understanding of the molecular basis of mitochondrial dynamics. It is now well established that mitochondria are highly dynamic organelles whose morphology, distribution, and activity can be regulated by fusion and fission. Several components of the core fusion and fission machinery have been identified, and experimental systems have been developed to understand their molecular mechanism of action.

In addition to this molecular understanding, genetic studies have revealed the importance of these processes in normal cell function, mammalian development, and human disease. Mitochondrial fusion is necessary for normal respiratory function in cells, and perturbations in fusion lead to developmental defects in mice and neurodegenerative disease in humans. This review emphasizes advances in our understanding of mitochondrial dynamics in mammals, although work in other systems is discussed when relevant.

\section{THE FUSION MACHINERY}

In mammalian cells, mitochondria take on a wide variety of shapes, ranging from long, interconnected tubules to individual, small spheres (Bereiter-Hahn \& Voth 1994). In cultured fibroblasts, the mitochondrial population consists mostly of short and long tubules, which constantly migrate along their long axes along microtubule tracks (Chen \& Chan 2004). These migrations are saltatory, consisting of movements back and forth along the tracks. In some cell types, the actin cytoskeleton is also used for transport (Hollenbeck \& Saxton 2005). While migrating along these tracks, a pair of mitochondria can encounter each other end to end and fuse. Even though an organized cytoskeleton is important as a scaffold for organizing the distribution and movement of mitochondria, it is not necessary for maintaining the tubular shape or fusion of mitochondria (Mattenberger et al. 2003).

In molecular genetic studies, an initial molecular inroad into a biological pathway can greatly accelerate the identification of additional genes. In the case of mitochondrial dynamics, it was the identification of the Drosophila mitofusin gene, Fzo, in sperm mitochondrial fusion (Hales \& Fuller 1997) that drove rapid advances in our knowledge of the molecular basis of mitochondrial fusion and fission. The yeast mitofusin homolog, Fzo1, was found to function similarly in mitochondrial fusion (Hermann et al. 1998, Rapaport et al. 1998), and genetic screens for extragenic suppressors of fusion mutants led to the identification of key molecules involved in mitochondrial fission (Fekkes et al. 2000, Mozdy et al. 2000, Tieu \& Nunnari 2000). Details of yeast mitochondrial dynamics can be found in other excellent reviews (Meeusen \& Nunnari 2005, Okamoto \& Shaw 2005, Shaw \& Nunnari 2002). Much of the fusion and fission machinery is conserved in mammals (Chen \& Chan 2004). 


\section{Mitofusins: Mfn1 and Mfn2}

Mitofusins are conserved, large GTPases localized to the mitochondrial outer membrane (Chen et al. 2003, Rojo et al. 2002, Santel \& Fuller 2001). In mammals, there are two closely related mitofusin homologs, Mfn 1 and Mfn2. Their essential role in mitochondrial fusion has been established through the generation of mice with targeted mutations (Chen et al. 2003). Cells lacking Mfn1 or Mfn2 have greatly reduced levels of mitochondrial fusion (Chen et al. 2005, Chen et al. 2003). Because of ongoing mitochondrial fission, the mitochondrial population in mutant cells is highly fragmented. In the absence of both Mfn 1 and Mfn2, there is absolutely no mitochondrial fusion, resulting in complete loss of mitochondrial tubules and poor mitochondrial function (Chen et al. 2005).

Mfn1 and Mfn2 appear to play similar roles in mitochondrial fusion. Both molecules are necessary for normal levels of mitochondrial fusion, but in cultured fibroblasts Mfn1 plays a more active role (Chen et al. 2005). Fibroblasts lacking Mfn1 have more severely fragmented mitochondria and show less residual mitochondrial fusion than do those lacking Mfn2. Mfn1 and Mfn2 can functionally replace each other. Cells lacking Mfn1 can be rescued by overexpression of Mfn2; conversely, cells lacking Mfn2 can be rescued by overexpression of Mfn1. Moreover, Mfn-null cells can be fully rescued by overexpression of either mitofusin. These results clearly indicate that the mitofusin homologs have very similar biochemical activities. It is likely that the cell-type-specific dependence for either Mfn1 or Mfn2, such as the requirement for Mfn2 in the placenta (Chen et al. 2003), is a consequence of their relative expression levels in different tissues. In spite of the functional similarities of Mfn1 and Mfn2, some differences in these molecules have been detected. OPA1 function shows a dependence on Mfn1 but not on Mfn2 (Cipolat et al. 2004), and Mfn1 shows more activity in a mitochondrial tethering assay (Ishihara et al. 2004).

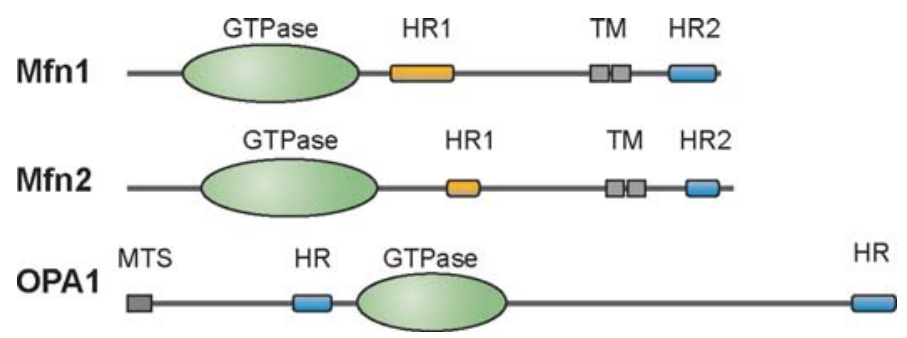

Figure 1

The mammalian mitochondrial fusion machinery. Schematic of Mfn1, Mfn2, and OPA1. All three proteins contain GTPase domains and hydrophobic heptad repeat (HR) regions. The transmembrane (TM) segments of the mitofusins are unusually long and contain charged residues that are thought to allow a U-turn in the mitochondrial outer membrane, so that almost the entire protein faces the cytosol (see Figure 2).

The membrane topology of mitofusins makes them good candidates for molecules involved in outer membrane fusion (Figure 1 and 2). Both the $\mathrm{N}$ - and $\mathrm{C}$-terminal regions of these proteins protrude from the mitochondrial outer membrane into the cytosol (Rojo et al. 2002). This topology implies a U-shaped transmembrane domain, and consistent with this, the transmembrane domain is unusually long and interrupted by several charged residues. The N-terminal domain of mitofusins contains a GTPase domain followed by a hydrophobic heptad repeat region, HR1. The C-terminal region contains a second hydrophobic heptad repeat region, HR2. As discussed below, the HR1 and HR2 regions likely play important roles in the fusion reaction.

\section{OPA1}

Mammalian OPA1 was initially identified as the gene mutated in autosomal dominant optic atrophy (DOA), a common cause of inherited visual loss (Alexander et al. 2000, Delettre et al. 2000). OPA1 and the yeast homolog Mgm1 are dynamin family GTPases that are located within the mitochondrial intermembrane space and are associated with the inner membrane (Griparic et al. 2004, Herlan et al. 2003, Olichon et al. 2002, Satoh et al. 2003, Wong et al. 2000) (Figures 1 and 2). The OPA1 gene produces multiple protein isoforms, owing to extensive alternative
Hydrophobic heptad repeat (HR): protein motif consisting of a seven-residue repeat, designated $a b c d e f g$, in which the $a$ and $d$ residues are primarily hydrophobic

DOA: dominant optic atrophy 


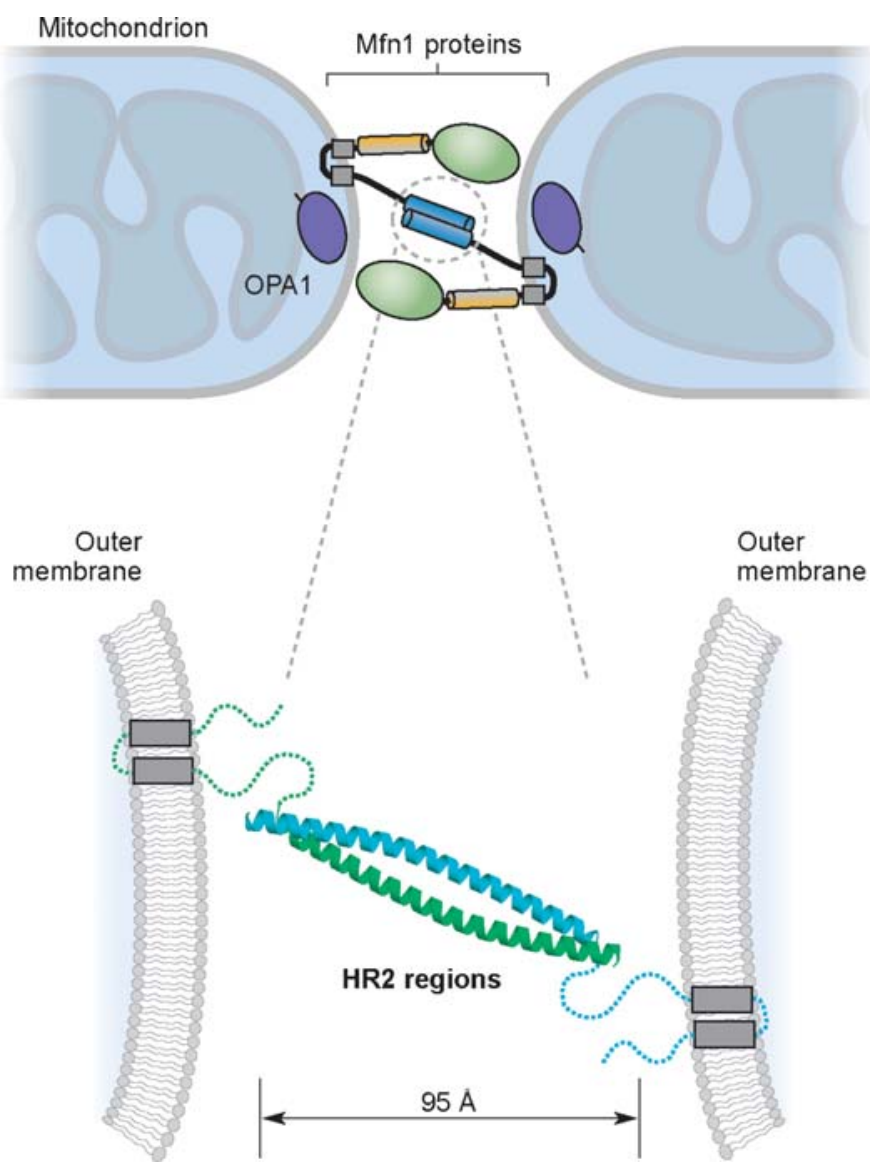

Figure 2

Mitochondrial tethering by Mfn1. Mfn1 is localized to the mitochondrial outer membrane, with most of the protein exposed to the cytosol. Homotypic interactions between Mfn 1 molecules spanning adjacent mitochondria are mediated by the HR2 region (blue cylinders) and result in tethering of the mitochondrial outer membranes. The HR2 crystal structure is shown below, indicating that the transmembrane domains are separated by the $95 \AA$ antiparallel coiled coil. The lower portion of this figure is adapted from Koshiba et al. 2004. Although events subsequent to tethering are unclear, note that the GTPase domains are in a position to modify the HR2 structure. OPA1 (purple oval), located in the intermembrane space and associated with the inner membrane, is the best candidate for direct involvement in inner membrane fusion.

MTS:

mitochondrial targeting sequence

RNAi: RNA interference splicing that yields eight mRNA isoforms (Delettre et al. 2001). In addition, by analogy with the yeast homolog Mgm1p (see below), OPA1 may be posttranslationally processed to yield additional isoforms.

Like mitofusins, OPA1 is essential for mitochondrial fusion. Knockdown of OPA1 by RNA interference (RNAi) leads to mitochon- drial fragmentation that is due to loss of mitochondrial fusion (Chen et al. 2005, Cipolat et al. 2004, Griparic et al. 2004). In addition to loss of fusion, OPA1 RNAi also leads to severe aberrations in cristae structure (Griparic et al. 2004, Olichon et al. 2003). Our understanding of OPA1 function is complicated by the fact that OPA1 overexpression can lead to mitochondrial fragmentation or elongation, depending on the experimental system (Chen et al. 2005, Cipolat et al. 2004, Griparic et al. 2004, Olichon et al. 2003).

Genetic and biochemical studies in yeast indicate that posttranslational processing of Mgm1p into two isoforms is important for its function, and a model for regulation of this processing has been proposed (Herlan et al. 2004). Unprocessed Mgm1p has an $\mathrm{N}$-terminal mitochondrial targeting sequence (MTS) followed by an extended hydrophobic region. The MTS targets the $\mathrm{N}$ terminus of $\mathrm{Mgm} 1 \mathrm{p}$ to the translocation machinery of mitochondrial inner membrane (TIM complex), where it is cleaved on the matrix side by the mitochondrial processing peptidase (MPP). After this cleavage, lateral exit from the TIM complex results in the formation of the long form of Mgm1p, 1-Mgm1p. If exit is delayed, the hydrophobic region of Mgm1p is pulled into the inner membrane, where it is further processed by the mitochondrial rhomboid protease, Rbd1p/Pcp1p, to form the short form of Mgm1p, s-Mgm1p (Herlan et al. 2003, 2004; McQuibban et al. 2003; Sesaki et al. 2003a). Therefore, the kinetics of sorting Mgm1p out of the TIM complex may determine the relative ratios of 1-Mgm1p and s-Mgm1p. The regulation of $\mathrm{Mgm} 1 \mathrm{p}$ processing is an important issue because the ratio of $1-M g m 1 p$ to s-Mgm1p affects mitochondrial dynamics and neither isoform by itself is sufficient for normal mitochondrial morphology. It will be interesting to determine whether these insights on Mgm1p processing apply to OPA1. It has been proposed that the mammalian ortholog of Rbd1p/Pcp1p, PARL, may be involved in OPA1 processing (McQuibban et al. 2003), 
but this proposal remains to be experimentally confirmed.

\section{THE FISSION MACHINERY}

Components of the mitochondrial fission pathway were first identified in yeast genetic screens (Bleazard et al. 1999, Fekkes et al. 2000, Mozdy et al. 2000, Tieu \& Nunnari 2000) and in studies of a dynamin-related protein in the worm (Labrousse et al. 1999). Extension of these studies into mammalian cells has identified dynamin-related protein 1 (Drp1) and Fis1 as components of the mammalian mitochondrial fission machinery (James et al. 2003, Smirnova et al. 2001, Yoon et al. 2003).

\section{Dynamin-Related Protein 1}

Drp1 is a key component of the mitochondrial fission machinery. Much of Drp1 is in the cytosol, but a subpool is localized to punctate spots on mitochondrial tubules, and a subset of these spots mark future sites of fission (Smirnova et al. 2001). Inhibition of Drp1 by expression of a dominant-negative mutant or by RNAi leads to increased length and interconnectivity of mitochondrial tubules, secondary to inhibition of fission (Lee et al. 2004, Smirnova et al. 2001). Drp1 contains several domains characteristic of dynamin family GTPases, including a GTPase domain, a central domain, and a GTPase effector domain (GED) (Figure 3).

\section{Fis1}

The other key component of the mammalian mitochondrial fission machinery is Fis1. Overexpression of Fis1 leads to mitochondrial fragmentation that is dependent on Drp1 (James et al. 2003, Yoon et al. 2003). Knockdown of Fis1 causes elongation of mitochondrial tubules, much as with inhibition of Drp1 (Lee et al. 2004).

Fis1 is a small protein that is uniformly localized to the outer membrane of mitochon-

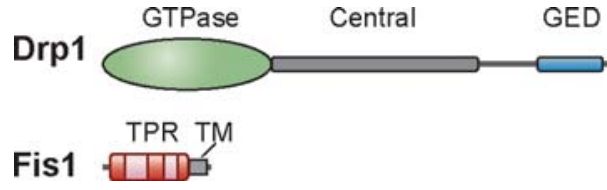

Figure 3

The mammalian mitochondrial fission machinery. Schematic of Drp1 and Fis1. Drp1 has domains typical of dynamin family GTPases, including a GTPase domain, a central domain, and a GTPase effector domain (GED). Fis1 is a small mitochondrial outer membrane protein whose $\mathrm{N}$-terminal region faces the cytosol (see Figure 4). This cytosolic domain ( $r e d$ and pink) forms a six-helix bundle that includes two central tetratricopeptide (TPR) repeats (pink).

dria through a single C-terminal transmembrane domain; most of the protein faces the cytosol (Figures 3 and 4). The cytosolic domain of Fis 1 consists of six antiparallel helices that form a helical bundle (Dohm et al. 2004; Suzuki et al. 2003, 2005). The central four helices consists of two tandem tetratricopeptide repeats (TPRs), a helix-turn-helix motif. One surface of the helical bundle is concave and is thought to act as a binding site for a small peptide ligand.

\section{OTHER PLAYERS}

Several other molecules have been proposed to regulate mitochondrial dynamics in mammals. Endophilin B1 is a BAR (Bin, amphiphysin, Rvs) domain-containing protein thought to be involved in mitochondrial fission (Karbowski et al. 2004b). BAR domains interact with lipid membranes and form crescent-shaped dimers with an intrinsic curvature (McMahon \& Gallop 2005). Because recombinant BAR domains can tubulate lipid membranes, the BAR domain may both sense membrane curvature as well as force curvature onto membranes. Knockdown of endophilin B1 by RNAi leads to a range of mitochondrial morphology abnormalities suggestive of a defect in fission (Karbowski et al. 2004b). Cells depleted for endophilin B1 show a mixture of tubules and spheres, often with unusual interconnections. Most strikingly, some cells
TIM: translocase of the inner membrane

MPP:

mitochondrial processing peptidase

Tetratricopeptide repeat (TPR): a protein motif consisting of two antiparallel $\alpha$-helices that is often multimerized to yield a protein-protein interaction module 
a

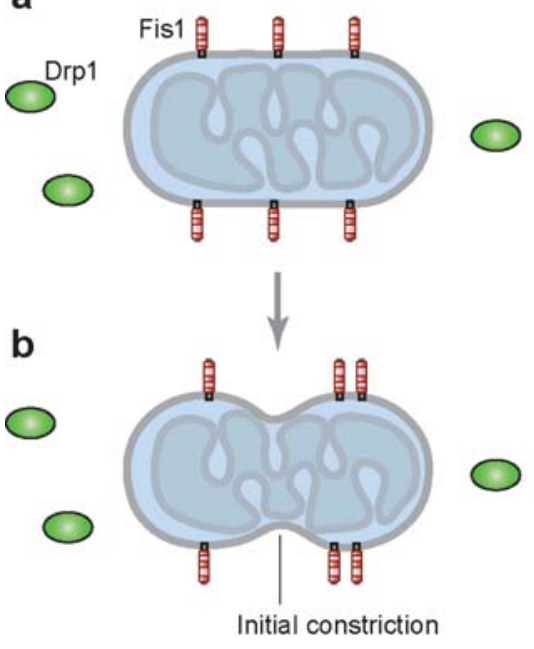

C

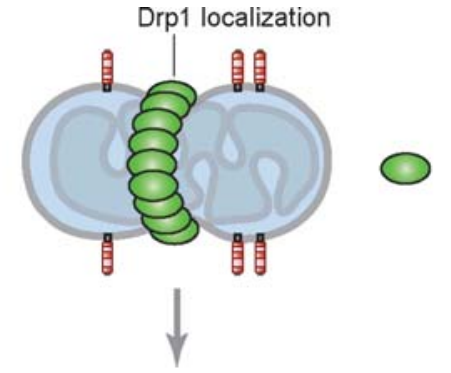

d

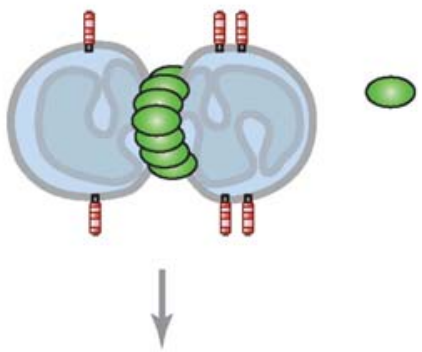

e

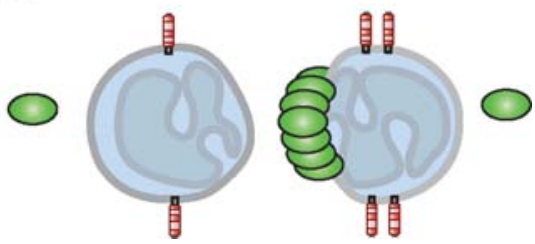

Figure 4

Mitochondrial fission by Fis1 and Drp1. (a) Fis1 resides uniformly on the mitochondrial outer membrane; most of the protein faces the cytosol. Drp1 (green ovals) exists both in the cytosol and on mitochondria in punctate spots (not shown). (b) Initial constriction of mitochondrial tubules occurs in a Drp1/Dnm1-independent manner. (c) In some cases, this constriction coincides with Drp1 localization, and $(d)$ Drp1 probably further constricts the mitochondrial tubule to mediate membrane fission. $(e)$ After fission is completed, the Drp1 complex is localized to the end of one of the daughter mitochondria prior to disassembly. Initial constriction of mitochondria and Drp1 localization are independent events; the diagrams are not meant to imply that they occur sequentially. Only a subset of Drp1 punctae on mitochondria proceeds to actual fission.

contain long, thin tubules that stain for outer membrane markers but not matrix markers. These observations indicate a dissociation of the mitochondrial outer membrane from the inner membrane and suggest that endophilin B1 may have a role in the control of outer membrane dynamics. However, the phenotypes generated by endophilin B1 depletion are distinct from the classic fission defects that occur in downregulation of Fis1 or Drp1, and it remains to be determined whether endophilin B1 is directly involved in mitochondrial fission.
The mitochondrial membrane protein MTP18 is another molecule that appears to play a role in mitochondrial fission (Tondera et al. 2005). The topology of this protein remains to be definitively determined, but the protein contains three putative transmembrane domains and is not exposed to the outside of mitochondria. Overexpression of MPT18 leads to mitochondrial fragmentation, and downregulation leads to mitochondrial elongation. It will be interesting to test whether endophilin B1 or MTP18 interacts with components of the fission machinery. 
GDAP (ganglioside-induced differentiation associated protein), Miro (mitochondrialRho-GTPase)-1, and Miro-2 are additional molecules that control mitochondrial morphology and are discussed below.

\section{MOLECULAR MECHANISM}

\section{Fusion Mechanism}

Studies on virus-mediated and SNAREmediated membrane fusion have highlighted some common themes in the fusion of biological membranes (Bonifacino \& Glick 2004, Eckert \& Kim 2001). First, the specificity of membrane fusion is ensured by the formation of specific protein complexes in trans between the donor and acceptor membranes. In the case of enveloped viruses, a subunit of the viral glycoprotein binds to a cell surface receptor on the host cell. In vesicle trafficking, the binding of Rab GTPases to their effector proteins provides initial specificity. This binding results in loose association of the two membrane compartments. In a subsequent step, formation of trans SNARE complexes between R- and Q-SNAREs on the two membranes provides an additional layer of specificity. Second, membrane merger is driven by formation of highly stable helical bundles. For example, in vesicle fusion, the formation of the trans SNARE complex results in a highly stable four-helix bundle that directly forces the donor and acceptor membrane bilayers into close proximity.

Mitochondrial fusion probably shares these two general features, and mitofusins appear to be involved in both. Mfn1 and Mfn2 are required for mitochondrial fusion, and they are located in the mitochondrial outer membrane, where they can initiate the interaction of mitochondria with each other. Importantly, mitofusins are required on adjacent mitochondria during the membrane fusion event (Koshiba et al. 2004). In mitochondrial fusion assays, mitochondria lacking mitofusins cannot fuse with mitochondria from wild-type cells. In yeast, Fzo1 is also

\section{MISSING COMPONENTS OF THE MAMMALIAN FUSION AND FISSION MACHINERY}

Several prominent components of the mitochondrial fusion and fission machinery (Ugo1, Mdv1, Caf4) identified in yeast do not have obvious counterparts in mammals. In the yeast mitochondrial fusion pathway, Fzo1 and Mgm1 both are physically associated with Ugo1, an outer membrane protein essential for fusion (Sesaki \& Jensen 2001, Sesaki et al. 2003b, Wong et al. 2003). It has been proposed that Ugo1 may coordinate fusion of the outer and inner membranes. In the fission pathway, two key yeast components are absent from the mammalian system. Mdv1 is a WD40 repeat-containing protein that is essential for mitochondrial fission (Shaw \& Nunnari 2002, Tieu \& Nunnari 2000). A WD40 repeat is a short, $\sim 40-$ residue motif, also called a WD or $\beta$-transducin repeat, that is multimerized to form a $\beta$-propeller structure that often serves as a scaffold for protein-protein interactions. Fis1 is localized uniformly on the mitochondrial outer membrane and works through Mdv1 and a related protein, Caf4, to recruit Dnm1 to punctate spots on mitochondria (Griffin et al. 2005). Mdv1 (Tieu et al. 2002) and Caf4 (Griffin et al. 2005) both physically interact with Fis1 and Dnm1, thereby allowing them to act as molecular adaptors in the assembly of the fission complex. In the absence of Mdv1 and Caf4, it is unclear how the mammalian fission machinery is assembled on the mitochondrial surface.

required on both mitochondria in vitro (Meeusen et al. 2004). Moreover, Mfn1 and Mfn2 form both homotypic and heterotypic complexes that can be detected by immunoprecipitation (Chen et al. 2003, Eura et al. 2003). Homotypic Mfn1 complexes, homotypic Mfn2 complexes, and heterotypic Mfn1Mfn2 complexes are all competent for fusion (Chen et al. 2005).

It is therefore likely that mitofusins form complexes in trans, that is, between adjacent mitochondria (Figure 3). Biochemical studies indicate that the $\mathrm{C}$-terminal region of Mfn1, containing the hydrophobic heptad repeat region HR2, can oligomerize with itself and with the analogous HR2 region from Mfn2. Structural studies indicate that
SNARE: soluble NSF attachment receptor 
Coiled coil: a

helical bundle consisting of 2-5 helices stabilized by hydrophobic interactions. It is formed by proteins with hydrophobic heptad repeats and is often used for protein oligomerization

Mitochondrial membrane potential: the electrical gradient that is present across the mitochondrial inner membrane and that is generated by proton pumping by components of the respiratory chain
Mfn1 HR2 forms a $95 \AA$, dimeric antiparallel coiled coil with transmembrane regions on either end (Koshiba et al. 2004). In a trans complex, such a structure would lead to close apposition of mitochondria but would still leave a significant gap. This structure is fundamentally different from the helical bundles formed during virus-mediated fusion and vesicle fusion, in which the membranespanning segments are forced into direct apposition. Therefore, the Mfn1 crystal structure likely represents a conformation of $\mathrm{Mfn} 1$ involved in the tethering of mitochondria, but not actual fusion. In vitro assays also support a role for Mfn1 in mitochondrial tethering (Ishihara et al. 2004). Fusion would require a conformational change that mediates closer apposition of mitochondrial membranes. Such a conformational change may occur by nucleotide-dependent changes in the GTPase domain or may involve other proteins.

Mitochondrial fusion involves mixing of matrix contents, indicating that both the outer and inner membranes must coordinately fuse. The coordination of outer and inner membrane fusion is a remarkable feat, given the complex geometry of the inner membrane. Extensive electron microscopic tomography has revealed that the internal structures of mitochondria are quite variable, depending on the cell type, and are more complicated than initial models have suggested (Frey \& Mannella 2000). A portion of the inner membrane (termed the inner boundary membrane) is positioned closely parallel to the outer membrane. However, the inner membrane has numerous invaginations (cristae) whose geometry can range from tubules to complex, interconnected plates. In general, these invaginations are connected to the inner boundary membrane by narrow tubules termed crista junctions. Given these geometric considerations, inner membrane fusion likely would occur at the inner boundary membrane because this part of the inner membrane lies closest to the outer membrane.
It is commonly assumed that outer and inner membrane fusion are tightly coordinated. However, by experimental manipulation, the two processes can be uncoupled, suggesting that they are mechanistically distinct. In an in vitro yeast mitochondrial fusion assay, outer membrane fusion required close association of mitochondria, low levels of GTP, and a proton gradient across the inner membrane (Meeusen et al. 2004). In contrast, progression to inner membrane fusion required additional GTP and an intact electrical gradient across the inner membrane. It is not known whether the requirement for membrane potential reflects a basic aspect of the fusion mechanism or whether it is secondary to structural effects on the inner membrane. In cultured mammalian cells, mitochondrial fusion is disrupted by $\mathrm{H}^{+}$ or $\mathrm{K}^{+}$ionophores that perturb the mitochondrial membrane potential (Ishihara et al. 2003, Legros et al. 2002, Mattenberger et al. 2003). Upon closer examination, it was found that these ionophores allow outer but not inner membrane fusion (Malka et al. 2005). Remarkably, such uncoupling can also be observed in untreated cells, suggesting that inner and outer membrane fusion may not be as tightly coordinated as commonly is assumed (Malka et al. 2005).

The machinery mediating inner membrane fusion is unknown, but it is likely that OPA1 plays a role because of its essential role in fusion (Chen et al. 2005, Cipolat et al. 2004) and association with the inner membrane (Griparic et al. 2004, Satoh et al. 2003). Although OPA1/Mgm1 are not exposed to the cytosol, cells lacking OPA1 or yeast lacking Mgm1 have no detectable outer membrane fusion (Chen et al. 2005, Sesaki et al. 2003b). At this point, it is not clear whether the defect in outer membrane fusion reflects an intrinsic role in that fusion event or rather a consequence of normal outer and inner membrane coupling. There are few clues to the molecular mechanism of OPA1. The homology of OPA1 to dynamin family GTPases suggests that it may be involved in controlling 
curvature or tubulation of the inner membrane, and indeed loss of OPA1 leads to severe defects in cristae structure (Griparic et al. 2004, Olichon et al. 2003).

\section{Fission Mechanism}

Although Fis1 and Drp1 are clearly required for mitochondrial fission, there is little known about their molecular mechanisms of action. A key unresolved issue is how Drp1 is recruited to mitochondria. Drp1 is present in both the cytosol and the mitochondria, with the mitochondrial pool localized to punctate spots (Smirnova et al. 2001). A subset of these spots becomes actual fission sites, and therefore the recruitment of Drp1 to mitochondria is a critical step in initiating the fission process. By analogy with yeast Dnm1, it would be expected that Drp1 localization to mitochondria is dependent on Fis1. However, efficient knockdown of Fis1 does not affect Drp1 recruitment (Lee et al. 2004).

Most insights about the mechanism of mitochondrial division have come from studies in yeast. Even in this system, it remains a mystery how Dnm1 becomes localized to discrete sites on mitochondria. Recruitment of Dnm1 depends on Fis1, which is uniformly localized to the mitochondrial outer membrane (Fekkes et al. 2000, Mozdy et al. 2000, Tieu \& Nunnari 2000). The cytosolic portion of Fis1 has a concave surface that acts as a binding surface (Karren et al. 2005, Suzuki et al. 2005). Mdv1 and Caf4 act as molecular adaptors by binding to Fis1 and Dnm1 (Griffin et al. 2005). Mdv1 appears to be able to exist both uniformly on mitochondria as well as in discrete spots that colocalize with Dnm1 spots (Cerveny et al. 2001, Griffin et al. 2005, Tieu \& Nunnari 2000). It is unclear how uniformly localized Fis1 can recruit Mdv1 or Dnm1 to discrete spots. One possibility is that Fis1 must become activated before it can recruit Dnm1 and that this activation occurs in a discontinuous manner. A second possibility is that additional factors other than Fis1/Mdv1/ Caf4 are used for recruitment of Dnm1. A low level of Dnm1 recruitment occurs in the absence of Fis1, implying an intrinsic affinity of Dnm1 for mitochondrial membranes. Perhaps Dnm1 is able to sense structural features on the mitochondrial membrane in addition to Mdv1 or Caf4 complexes.

It remains to be resolved how Drp1/Dnm1 mediate fission once recruited to mitochondria. Because Drp1 is a dynamin family GTPase, it is thought to act as a mechanochemical enzyme that uses GTP hydrolysis to drive constriction at mitochondrial fission sites (Shaw \& Nunnari 2002, Smirnova et al. 2001). This mode of action is analogous to proposed roles of classical dynamin in constriction of invaginating vesicle necks during clathrin-mediated endocytosis (Praefcke \& McMahon 2004). Indeed, Dnm1 in vitro shows assembly-dependent GTP hydrolysis and is able to assemble into spirals with dimensions consistent with constricted mitochondrial tubules (Ingerman et al. 2005). Recombinant human Drp1 can tubulate artificial liposomes (Yoon et al. 2001). Only a small subset of Dnm1 punctae progresses to actual fission (Legesse-Miller et al. 2003), and progression probably depends on factors controlling the assembly and activation of Dnm 1 . Mdv1 is likely one such factor because recruitment of Dnm1 to mitochondria in the absence of Mdv1 fails to stimulate fission (Cerveny \& Jensen 2003, Fekkes et al. 2000, Tieu \& Nunnari 2000, Tieu et al. 2002).

However, the model that Drp1/Dnm1mediated constriction drives fission is complicated by the observation that transient constrictions commonly occur on yeast mitochondrial tubules in the absence of Dnm1, indicating that the initial steps of fission are Dnm1 independent (Legesse-Miller et al. 2003). Studies of mitochondrial fission in the red alga Cyanidioschyzon merolae also support a late role for Drp1. In C. merolae, the fission machinery consists of not only a dynamin-like protein, CmDnm1, but also the extra component CmFtsZ1, an ortholog of the bacterial division protein FtsZ (Nishida et al. 2003). CmFtsZ1 is the first marker of mitochondrial 
fission, forming a ring on the matrix side of the mitochondrial inner membrane before any constriction has occurred. After initial constriction, CmDnm1 is recruited to the cytosolic surface of the outer membrane, where it is thought to complete the severing of membranes. This mode of mitochondrial division resembles chloroplast division in plants, which involves proteins similar to FtsZ and Drp1 acting in a similar order (Osteryoung \& Nunnari 2003). In these cases, the Drp1-like molecules act late in organelle division.

FtsZ orthologs are also involved in mitochondrial fission in the alga Mallomonas splendens and in the amoeba Dictyostelium discoideum, but no mitochondrial Drp1-like molecules have been found (Beech et al. 2000, Gilson et al. 2003, Kiefel et al. 2004). Thus, although most organisms use Drp1 proteins to divide mitochondria, there are a few organisms that use FtsZ-like proteins alone to divide mitochondria and at least one known organism (C. merolae) that uses both FtsZand Drp1-like proteins. In cases in which only Drp1 is used, it is not known whether a separate protein has replaced the FtsZ-like function in constriction of the inner membrane or whether only constriction at the outer membrane by Drp1 is necessary. In support of the former possibility, imaging studies in yeast indicate that matrix separations, presumably reflecting inner membrane remodeling, occur in the absence of Fis1 or Dnm1 (Jakobs et al. 2003). In addition, under certain drug treatments, some mammalian cells similarly can show inner membrane fission without outer membrane fission (Duncan et al. 1980).

Mitochondrial fission likely is coordinated with other cellular processes. The mitochondrial genome is organized into discrete structures termed nucleoids, and fission generally results in daughter mitochondria that each contains at least one nucleoid, even in cells with highly fragmented mitochondria (Legros et al. 2004, Margineantu et al. 2002). This observation suggests that fission site determination may somehow be linked to nucleoid position. Mitochondrial fission may also be linked to the cell cycle. Morphological studies indicate that cultured mammalian cells in late $\mathrm{S}$ and $M$ phases typically have fragmented mitochondria (Barni et al. 1996, Margineantu et al. 2002). Therefore, cells may divide their mitochondria to facilitate segregation during cell division. However, studies in yeast confirm that mitochondrial inheritance can occur in the absence of mitochondrial fission (Bleazard et al. 1999, Sesaki \& Jensen 1999).

The roles of Fis1 and Drp1 in organelle division are not limited to mitochondrial fission. Both proteins also localize to peroxisomes, and knockdown of either leads to elongation of peroxisomes (Koch et al. 2005). As with mitochondria, constriction of peroxisomes, but not fission, can occur in the absence of Drp1 (Koch et al. 2004).

\section{BIOLOGICAL SIGNIFICANCE}

\section{Cell Biology of Fusion and Fission}

Several assays have been developed to measure mitochondrial fusion directly. The most common method involves artificially fusing cells with polyethylene glycol (PEG) or a virus. If two parental cells containing fluorescently labeled mitochondria are fused, mitochondrial fusion can be quantified in the cell hybrids by scoring for colocalization of fluorophores (Chen et al. 2003, Ishihara et al. 2003, Legros et al. 2002, Mattenberger et al. 2003). In such experiments, extensive mitochondrial fusion is detectable a few hours after cell fusion and is usually complete by $8 \mathrm{~h}$. This method is conceptually similar to the zygotic assay used to analyze mitochondrial fusion in yeast (Nunnari et al. 1997). Mitochondrial fusion can also be quantified through the use of a mitochondrially localized, photoactivable GFP (Karbowski et al. 2004a). Mitochondria in cells expressing such a construct are only weakly fluorescent, and a laser can be used to photoactivate the GFP in a small subset of mitochondria. The diffusion of the activated GFP to neighboring mitochondria is a measure of mitochondrial fusion. Mammalian 
mitochondrial fusion has yet to be reconstituted in vitro, although such an assay has been developed in yeast (Meeusen et al. 2004).

The morphology of mitochondria depends on the balance between the opposing processes of fusion and fission. Unbalanced fission leads to fragmentation, and unbalanced fusion leads to elongation. In cells with reduced mitochondrial fusion, mitochondrial tubules can be restored by simultaneous inhibition of fission (Chen et al. 2003). In normal development, the control of these processes can change the shape of mitochondria to suit a particular developmental function. For example, developmental upregulation of Fzo during Drosophila spermatogenesis leads to fusion of mitochondria to form the Nebenkern structure, which is important for male fertility (Hales \& Fuller 1997).

In addition to the control of mitochondrial shape, fusion and fission are also important for the bioenergetic function of mitochondria. In cells lacking mitofusins or OPA1, mitochondrial function is greatly diminished (Chen et al. 2005). The mitochondrial population within individual cells shows heterogeneity in membrane potential, and oxygen consumption is compromised. As a result, cells without mitochondrial fusion grow much more slowly than their wild-type counterparts. Other genetic manipulations that lead to mitochondrial fragmentation do not lead to such functional changes, and therefore fusion per se, apart from mitochondrial shape, appears to be important for mitochondrial function.

Why mitochondrial fusion is so important remains to be understood. It is probably because mitochondrial fusion allows mitochondria within a cell to cooperate with each other (Chen et al. 2003). In a wild-type cell, high rates of fusion and fission constantly change the identity of individual mitochondria. A discrete mitochondrion at one point in time will be changed at a later time by the addition of new mitochondrial material through fusion or by the removal of material through division. Therefore, mitochondria are not autonomous organelles; during their lifetime, their boundaries are constantly redefined. In cells lacking mitochondrial fusion, mitochondria become autonomous organelles that are unable to interact with their neighbors. In this case, defects in mitochondrial function-perhaps due to depletion of substrates, mitochondrial DNA (mtDNA), or metabolites-cannot be complemented by fusion with healthy mitochondria.

Mitochondrial fusion can also protect cells from the detrimental effects of mtDNA mutations by allowing functional complementation of mtDNA gene products. Cell hybrids formed by fusing parental cells carrying different pathogenic mtDNA mutations have restored respiratory activity (Ono et al. 2001). For unknown reasons, this complementation does not occur until approximately two weeks after cell fusion, even though in PEG fusion assays, mitochondrial fusion can be detected within a few hours. In animal models of mtDNA disease, tissues do not show defective mitochondria until the load of pathogenic mtDNA is very high (Nakada et al. 2001). Significantly, in cells with less than $60 \%$ pathogenic mtDNA, all the mitochondria are functional, indicating that fusion protects the function of the mitochondrial population.

\section{Mitochondrial Dynamics in Development and Apoptosis}

Mitochondrial fusion is an essential process in mammals. Mice lacking either Mfn1 or Mfn2 die in utero at approximately embryonic day 10.5-11.5 (Chen et al. 2003). Mfn2 mutant embryos develop normally until placental insufficiency leads to retardation of growth. In the absence of $\mathrm{Mfn} 2$, the trilaminar structure of the placenta is disrupted. The resulting placenta has very few trophoblast giant cells, polyploid cells that provide a number of cellular functions necessary for maintenance of the embryo.

Although these mutant mice could not be used to study later developmental functions
mtDNA:

mitochondrial DNA 
of mitofusins, it is likely that mitochondrial fusion also plays important roles in adult cells. In several tissues, ultrastructural studies of mitochondrial morphology during tissue differentiation indicate that mitochondria undergo dramatic morphological transitions, including increases in tubule length and interconnectivity that are likely due to developmentally regulated fusion (Chen \& Chan 2004). Because neurodegenerative diseases can be caused by mutations in human Mfn2 or OPA1 (Alexander et al. 2000, Delettre et al. 2000, Zuchner et al. 2004), certain neurons likely are particularly vulnerable to perturbations in mitochondrial dynamics. In cultures of hippocampal neurons, mitochondria appear to be recruited into actively growing dendritic protrusions, particularly when the neurons are activated by depolarization ( $\mathrm{Li}$ et al. 2004). Perturbation of mitochondrial dynamics in this system leads to a reduction in dendritic spine growth and synapse formation, suggesting that regulated recruitment of mitochondria is important for dendritic structure and plasticity.

Studies of Drosophila mutants have provided clues as to why mitochondria are important to neurons. Mutations in Drp1 or Miro (mitochondrial-Rho-GTPase) result in drastic reductions in the number of mitochondria that can reach nerve terminals (Guo et al. 2005, Verstreken et al. 2005). Miro is a mitochondrial outer membrane protein that contains tandem GTPase domains and EF hands, $\mathrm{Ca}^{2+}$-binding motifs. Mammals contain two homologs, Miro-1 and Miro-2, that regulate mitochondrial morphology (Fransson et al. 2003). The yeast ortholog, Gem1, also regulates mitochondrial morphology through a pathway separate from mitochondrial fusion or fission (Frederick et al. 2004). In the case of Drosophila Miro mutants, the absence of mitochondria from the neuromuscular junction leads to structural defects in the synapse (Guo et al. 2005). For both Miro and Drp1 mutants, the neurons show only modest defects in $\mathrm{Ca}^{2+}$ buffering that are aggravated by prolonged, high-intensity activity (Guo et al.
2005, Verstreken et al. 2005). The primary defect appears to be the reduced amount of ATP available at the nerve terminal.

Although mouse mutants of mitochondrial fission genes have not been reported, it is likely that mitochondrial fission is also an essential function. In Caenorbabditis elegans, knockdown of Drp1 by RNAi leads to very early embryonic lethality (Labrousse et al. 1999). In addition, mitochondrial fission plays an important role in apoptosis. In several models of apoptosis, mitochondria fragment at the onset of cell death (Breckenridge et al. 2003, Desagher \& Martinou 2000, Frank et al. 2001). This fragmentation occurs at approximately the time that Bax, a proapoptotic $\mathrm{Bcl} 2$ family member, translocates to mitochondria, and before caspase activation. Fragmentation depends on the activity of Drp1 and Fis1, and inhibition of these molecules reduces the level of apoptosis (Breckenridge et al. 2003, Frank et al. 2001, Lee et al. 2004). These events are likely to be relevant for apoptotic events in vivo. In C. elegans, developmentally regulated apoptosis also results in mitochondrial fragmentation that is Drp1 dependent (Jagasia et al. 2005). Blockage of Drp1 function reduces the efficiency of cell death. However, the proapoptotic role of mitochondrial fission in cell death is not universal. In ceramideinduced cell death, $\mathrm{Ca}^{2+}$ is a key effector that moves from the endoplasmic reticulum to mitochondria, ultimately leading to mitochondrial permeabilization and caspase activation. This $\mathrm{Ca}^{2+}$-mediated signaling is enhanced in cells with interconnected mitochondria, and therefore forced fragmentation of mitochondria leads to protection from cell death (Szabadkai et al. 2004).

Whereas mitochondrial fission often plays a proapoptotic role, mitochondrial fusion seems to protect cells from cell death. Mitochondrial fusion is reduced following induction of apoptosis (Karbowski et al. 2004a), and overexpression of mitofusins can reduce the level of apoptosis (Sugioka et al. 2004). Depletion of OPA1 or mitofusins results in poor cell growth (Chen et al. 2005) and enhances 
susceptibility to apoptotic stimuli (Lee et al. 2004, Olichon et al. 2003, Sugioka et al. 2004).

\section{Mitochondrial Dynamics in Disease}

Mutations in Mfn2 cause Charcot-MarieTooth subtype 2A (CMT2A), a peripheral neuropathy characterized by muscle weakness and sensory loss in the distal limbs (Zuchner et al. 2004). The most common form of CMT (CMT1) involves defects in the Schwann cells that myelinate the peripheral nerves (Kuhlenbaumer et al. 2002, Zuchner \& Vance 2005). As a result of Schwann cell dysfunction, patients have reduced nerve conduction velocities and show demyelination in nerve biopsies. In contrast, patients with CMT2A have normal nerve conduction velocities, and the peripheral nerve defect is thought to be axonal, or intrinsic to the motor and sensory neurons. Despite the pathological distinctions between the two subtypes, the symptoms are similar and consist of weakness in the feet, calves, hands, and forearms. Foot weakness causes footdrop and a characteristic high gait to compensate. Long-term imbalance of foot muscles leads to an abnormally high arch and hyperextension of the toes, a foot deformity termed pes cavus, or claw foot. Another axonal type of CMT with associated optic atrophy has also been linked to mutations in Mfn2 (Zuchner et al. 2006).

Most of the Mfn2 mutations causing CMT2A reside in or near the GTPase domain (Kijima et al. 2005, Lawson et al. 2005, Zuchner et al. 2004). Because the GTPase domain is essential for Mfn2 function, CMT2A may be due to haploinsufficiency of Mfn2 (Zuchner et al. 2004). However, mice heterozygous for Mfn2 do not have neuromuscular defects (Chen et al. 2003). It is unclear why long motor and sensory neurons are specifically affected in CMT2A, given that Mfn2 is broadly expressed in adult tissues. Hopefully these issues can be addressed through animal models.

GDAP1, the gene mutated in CMT4A, may also be involved in the control of mi- tochondrial dynamics (Niemann et al. 2005). Patients with CMT4A exhibit both demyelination and axonal neuropathy, and consistent with this mixed presentation, GDAP1 is expressed in both Schwann cells and neurons. GDAP1 contains two glutathione-Stransferase domains and is localized to the outer membrane of mitochondria. Overexpression of GDAP1 leads to mitochondrial fragmentation that is dependent on Drp1, implicating involvement of the normal fission machinery. Reduction of GDAP1 expression leads to elongation of mitochondrial tubules, suggesting that GDAP1 is involved in mitochondrial fission. Disease alleles of GDAP1 either prevent mitochondrial localization or reduce the mitochondrial fragmentation activity of GDAP1.

The most common form of inherited optic atrophy is autosomal dominant optic atrophy (DOA), in which patients exhibit bilateral optic nerve atrophy due to loss of retinal ganglion cells (Delettre et al. 2002). Several genetic loci have been linked to this disease; the OPA1 is by far the most commonly affected gene (Alexander et al. 2000, Delettre et al. 2000). In one OPA1 pedigree, monocytes from patients were found to have a defect in mitochondrial distribution, with mitochondria that clumped instead of being dispersed within the cell (Delettre et al. 2000). Unfortunately, ultrastructural studies of mitochondria in retinal ganglion cells from patient samples have not been reported. However, knockdown of OPA1 in cultured retinal ganglion cells also leads to aggregation of mitochondria (Kamei et al. 2005). As expected, OPA1 is expressed in the retina, although it remains unclear whether it is broadly expressed there or restricted to retinal ganglion cells (Ju et al. 2005, Kamei et al. 2005, Pesch et al. 2004).

Given the autosomal dominant nature of DOA, and the observation that some OPA1 disease alleles are severely truncated, haploinsufficiency of OPA1 likely accounts for some cases of DOA (Delettre et al. 2002). Most OPA1 disease alleles, however,
CMT: Charcot-

Marie-Tooth

disease 
contain less severe truncations or have point mutations in the GTPase or central domain (http://lbbma.univ-angers.fr/ eOPA1/). In such cases, it remains to be determined whether these alleles have dominantnegative effects on the remaining wild-type allele. Other dynamin family GTPases have been shown to assemble into oligomers, and mutants with truncations or point mutations often have dominant-negative activity (Praefcke \& McMahon 2004). Clarification of these issues has been hampered by the lack of a straightforward cell-based system for structure/function analysis of OPA1.

It has been suggested, on the basis of the known role of OPA1 in regulation of mitochondrial dynamics, that OPA1-type DOA is a mitochondrial disease (Delettre et al. 2002). Recently, there has been evidence that this disease may have additional parallels to classic mitochondrial encephalomyopathy. In some pedigrees of DOA caused by OPA1 mutations, there is associated neurosensory hearing loss, ptosis, and ophthalmoplegia (Payne et al. 2004). Given the frequent occurrence of myopathy in mitochondrial disorders, it would be interesting to determine whether the ptosis and ophthalmoplegia are due to muscle defects. Intriguingly, metabolic studies of OPA1-type DOA patients suggest a deficit in mitochondrial function within skeletal muscle (Lodi et al. 2004).

\section{PERSPECTIVES}

Although it was an obscure subject a few years ago, mitochondrial dynamics has emerged as a fundamental cell biological process with clearly important roles in mammalian biology. With key components of the fusion and fission machinery in hand, biologists have begun to make advances in understanding the molecular mechanisms of these membrane remodeling events. To make further progress, detailed structural and biochemical knowledge will be required.

Mitochondrial dynamics is important not only for control of the shape but also the function of mitochondria. We must devise new methods to understand how mitochondria interact with each other and why such interactions are important for the health of the aggregate mitochondrial population within a cell. An appreciation of this feature of mitochondrial biology will be important for understanding why certain cell types, for example neurons, are particularly dependent on mitochondrial dynamics. Given that perturbations in mitochondrial fusion result in neurodegenerative disease, such approaches hold promise for the improvement of human health.

\section{SUMMARY POINTS}

1. Mitochondria are dynamic organelles whose morphologies are controlled by fusion and fission.

2. Mitochondrial fusion and fission are essential for normal mitochondrial function, implying that mitochondria do not function well as autonomous organelles.

3. The mitofusins Mfn1 and Mfn2, key components of the mammalian mitochondrial fusion machinery, are localized to the outer membrane and mediate tethering of mitochondria during the fusion process.

4. The mammalian mitochondrial fission machinery consists of the outer membrane protein Fis1 and the dynamin-related GTPase Drp1.

5. Mitochondrial dynamics plays important roles in vertebrate development and programmed cell death. 
6. Mutations in the mitochondrial fusion machinery lead to two human neurodegenerative disorders, Charcot-Marie-Tooth subtype 2A and autosomal dominant optic atrophy.

\section{FUTURE ISSUES}

1. After mitochondrial tethering by mitofusins, how is membrane merger accomplished? Membrane fusion likely involves rearrangement of the mitofusin tether to force closer membrane apposition.

2. How is inner membrane fusion coupled to outer membrane fusion?

3. How is inner membrane structure controlled?

4. Can in vitro reconstitution be developed for mammalian mitochondrial fusion?

5. Are there functional equivalents for Ugo1, Caf4, and Mdv1 in mammals?

6. How is OPA1 posttranslationally processed? By analogy with yeast Mgm1, it is likely that processing by a mitochondrial protease is important for the regulation of OPA1 function.

7. How is Drp1 recruited to mitochondria into punctate spots, and how do some of these proceed to actual fission?

8. Studies of cells lacking mitofusins or OPA1 indicate that autonomous mitochondria do not function well. Why do mitochondria need to exchange contents?

9. What is the basis for the extreme tissue specificity of CMT2A and DOA, given the widespread expression of Mfn2 and OPA1?

10. Why is mitochondrial dynamics so important in neurons?

\section{ACKNOWLEDGMENTS}

I am grateful to Hsiuchen Chen for helpful comments on the manuscript. Work in my laboratory is supported by the NIH (GM062967), the Muscular Dystrophy Association, and the United Mitochondrial Disease Foundation.

\section{LITERATURE CITED}

Alexander C, Votruba M, Pesch UE, Thiselton DL, Mayer S, et al. 2000. OPA1, encoding a dynamin-related GTPase, is mutated in autosomal dominant optic atrophy linked to chromosome 3q28. Nat. Genet. 26:211-15

Barni S, Sciola L, Spano A, Pippia P. 1996. Static cytofluorometry and fluorescence morphology of mitochondria and DNA in proliferating fibroblasts. Biotech. Histochem. 71:66-70

Beech PL, Nheu T, Schultz T, Herbert S, Lithgow T, et al. 2000. Mitochondrial FtsZ in a chromophyte alga. Science 287:1276-79

Bereiter-Hahn J, Voth M. 1994. Dynamics of mitochondria in living cells: shape changes, dislocations, fusion, and fission of mitochondria. Microsc. Res. Tech. 27:198-219 


\section{Evidence that mitochondrial fusion affects not only the shape but also the function of mitochondria.}

The first clear indication that Drp1, a component of the mitochondrial fission machinery, affects apoptosis.
Bleazard W, McCaffery JM, King EJ, Bale S, Mozdy A, et al. 1999. The dynamin-related GTPase Dnm1 regulates mitochondrial fission in yeast. Nat. Cell Biol. 1:298-304

Bonifacino JS, Glick BS. 2004. The mechanisms of vesicle budding and fusion. Cell 116:153-66

Breckenridge DG, Stojanovic M, Marcellus RC, Shore GC. 2003. Caspase cleavage product of BAP31 induces mitochondrial fission through endoplasmic reticulum calcium signals, enhancing cytochrome $\mathrm{c}$ release to the cytosol. F. Cell Biol. 160:1115-27

Cerveny KL, Jensen RE. 2003. The WD-repeats of Net2p interact with Dnm1p and Fis1p to regulate division of mitochondria. Mol. Biol. Cell 14:4126-39

Cerveny KL, McCaffery JM, Jensen RE. 2001. Division of mitochondria requires a novel DMN1-interacting protein, Net2p. Mol. Biol. Cell 12:309-21

Chen H, Chan DC. 2004. Mitochondrial dynamics in mammals. Curr. Top. Dev. Biol. 59:119-44

Chen H, Chomyn A, Chan DC. 2005. Disruption of fusion results in mitochondrial heterogeneity and dysfunction. F. Biol. Chem. 280:26185-92

Chen H, Detmer SA, Ewald AJ, Griffin EE, Fraser SE, Chan DC. 2003. Mitofusins Mfn1 and Mfn2 coordinately regulate mitochondrial fusion and are essential for embryonic development. F. Cell Biol. 160:189-200

Cipolat S, Martins de Brito O, Dal Zilio B, Scorrano L. 2004. OPA1 requires mitofusin 1 to promote mitochondrial fusion. Proc. Natl. Acad. Sci. USA 101:15927-32

Delettre C, Griffoin JM, Kaplan J, Dollfus H, Lorenz B, et al. 2001. Mutation spectrum and splicing variants in the OPA1 gene. Hum. Genet. 109:584-91

Delettre C, Lenaers G, Griffoin JM, Gigarel N, Lorenzo C, et al. 2000. Nuclear gene OPA1, encoding a mitochondrial dynamin-related protein, is mutated in dominant optic atrophy. Nat. Genet. 26:207-10

Delettre C, Lenaers G, Pelloquin L, Belenguer P, Hamel CP. 2002. OPA1 (Kjer type) dominant optic atrophy: a novel mitochondrial disease. Mol. Genet. Metab. 75:97-107

Desagher S, Martinou JC. 2000. Mitochondria as the central control point of apoptosis. Trends Cell Biol. 10:369-77

Dohm JA, Lee SJ, Hardwick JM, Hill RB, Gittis AG. 2004. Cytosolic domain of the human mitochondrial fission protein fis1 adopts a TPR fold. Proteins 54:153-56

Duncan CJ, Greenaway HC, Publicover SJ, Rudge MF, Smith JL. 1980. Experimental production of "septa" and apparent subdivision of muscle mitochondria. F. Bioenerg. Biomembr. $12: 13-33$

Eckert DM, Kim PS. 2001. Mechanisms of viral membrane fusion and its inhibition. Annu. Rev. Biochem. 70:777-810

Eura Y, Ishihara N, Yokota S, Mihara K. 2003. Two mitofusin proteins, mammalian homologues of $\mathrm{FZO}$, with distinct functions are both required for mitochondrial fusion. F. Biochem. 134:333-44

Fekkes P, Shepard KA, Yaffe MP. 2000. Gag3p, an outer membrane protein required for fission of mitochondrial tubules. F. Cell Biol. 151:333-40

Frank S, Gaume B, Bergmann-Leitner ES, Leitner WW, Robert EG, et al. 2001. The role of dynamin-related protein 1, a mediator of mitochondrial fission, in apoptosis. Dev. Cell 1:515-25

Fransson A, Ruusala A, Aspenstrom P. 2003. Atypical Rho GTPases have roles in mitochondrial homeostasis and apoptosis. 7. Biol. Chem. 278:6495-502

Frederick RL, McCaffery JM, Cunningham KW, Okamoto K, Shaw JM. 2004. Yeast Miro GTPase, Gem1p, regulates mitochondrial morphology via a novel pathway. F. Cell Biol. 167:87-98

Frey TG, Mannella CA. 2000. The internal structure of mitochondria. Trends Biochem. Sci. 25:319-24 
Gilson PR, Yu XC, Hereld D, Barth C, Savage A, et al. 2003. Two Dictyostelium orthologs of the prokaryotic cell division protein FtsZ localize to mitochondria and are required for the maintenance of normal mitochondrial morphology. Eukaryot. Cell 2:1315-26

Griffin EE, Graumann J, Chan DC. 2005. The WD40 protein Caf4p is a component of the mitochondrial fission machinery and recruits Dnm1p to mitochondria. 7. Cell Biol. 170:237-48

Griparic L, van der Wel NN, Orozco IJ, Peters PJ, van der Bliek AM. 2004. Loss of the intermembrane space protein Mgm1/OPA1 induces swelling and localized constrictions along the lengths of mitochondria. F. Biol. Chem. 279:18792-98

Guo X, Macleod GT, Wellington A, Hu F, Panchumarthi S, et al. 2005. The GTPase dMiro is required for axonal transport of mitochondria to Drosophila synapses. Neuron 47:379-93

Hales KG, Fuller MT. 1997. Developmentally regulated mitochondrial fusion mediated by a conserved, novel, predicted GTPase. Cell 90:121-29

Herlan M, Bornhovd C, Hell K, Neupert W, Reichert AS. 2004. Alternative topogenesis of Mgm1 and mitochondrial morphology depend on ATP and a functional import motor. 7. Cell Biol. 165:167-73

Herlan M, Vogel F, Bornhovd C, Neupert W, Reichert AS. 2003. Processing of Mgm1 by the rhomboid-type protease Pcp1 is required for maintenance of mitochondrial morphology and of mitochondrial DNA. F. Biol. Chem. 278:27781-88

Hermann GJ, Thatcher JW, Mills JP, Hales KG, Fuller MT, et al. 1998. Mitochondrial fusion in yeast requires the transmembrane GTPase Fzo1p. F. Cell Biol. 143:359-73

Hollenbeck PJ, Saxton WM. 2005. The axonal transport of mitochondria. F. Cell Sci. 118:541119

Ingerman E, Perkins EM, Marino M, Mears JA, McCaffery JM, et al. 2005. Dnm1 forms spirals that are structurally tailored to fit mitochondria. F. Cell Biol. 170:1021-27

Ishihara N, Eura Y, Mihara K. 2004. Mitofusin 1 and 2 play distinct roles in mitochondrial fusion reactions via GTPase activity. F. Cell Sci. 117:6535-46

Ishihara N, Jofuku A, Eura Y, Mihara K. 2003. Regulation of mitochondrial morphology by membrane potential, and DRP1-dependent division and FZO1-dependent fusion reaction in mammalian cells. Biochem. Biophys. Res. Commun. 301:891-98

Jagasia R, Grote P, Westermann B, Conradt B. 2005. DRP-1-mediated mitochondrial fragmentation during EGL-1-induced cell death in C. elegans. Nature 433:754-60

Jakobs S, Martini N, Schauss AC, Egner A, Westermann B, Hell SW. 2003. Spatial and temporal dynamics of budding yeast mitochondria lacking the division component Fis1p. 7. Cell Sci. 116:2005-14

James DI, Parone PA, Mattenberger Y, Martinou JC. 2003. hFis1, a novel component of the mammalian mitochondrial fission machinery. 7. Biol. Chem. 278:36373-79

Ju WK, Misaka T, Kushnareva Y, Nakagomi S, Agarwal N, et al. 2005. OPA1 expression in the normal rat retina and optic nerve. F. Comp. Neurol. 488:1-10

Kamei S, Chen-Kuo-Chang M, Cazevieille C, Lenaers G, Olichon A, et al. 2005. Expression of the Opa1 mitochondrial protein in retinal ganglion cells: Its downregulation causes aggregation of the mitochondrial network. Invest. Ophthalmol. Vis. Sci. 46:4288-94

Karbowski M, Arnoult D, Chen H, Chan DC, Smith CL, Youle RJ. 2004a. Quantitation of mitochondrial dynamics by photolabeling of individual organelles shows that mitochondrial fusion is blocked during the Bax activation phase of apoptosis. F. Cell Biol. 164:493-99

Karbowski M, Jeong SY, Youle RJ. 2004b. Endophilin B1 is required for the maintenance of mitochondrial morphology. 7. Cell Biol. 166:1027-39
A study of the role of mitochondria in the neuromuscular junction. 
Evidence that mitofusins mediate mitochondrial tethering during fusion.

These authors developed an in vitro assay to study mitochondrial fusion.
Karren MA, Coonrod EM, Anderson TK, Shaw JM. 2005. The role of Fis1p-Mdv1p interactions in mitochondrial fission complex assembly. F. Cell Biol. 171:291-301

Kiefel BR, Gilson PR, Beech PL. 2004. Diverse eukaryotes have retained mitochondrial homologues of the bacterial division protein FtsZ. Protist 155:105-15

Kijima K, Numakura C, Izumino H, Umetsu K, Nezu A, et al. 2005. Mitochondrial GTPase mitofusin 2 mutation in Charcot-Marie-Tooth neuropathy type 2A. Hum. Genet. 116:2327

Koch A, Schneider G, Luers GH, Schrader M. 2004. Peroxisome elongation and constriction but not fission can occur independently of dynamin-like protein 1. F. Cell Sci. 117:39954006

Koch A, Yoon Y, Bonekamp NA, McNiven MA, Schrader M. 2005. A role for Fis1 in both mitochondrial and peroxisomal fission in mammalian cells. Mol. Biol. Cell 16:5077-86

Koshiba T, Detmer SA, Kaiser JT, Chen H, McCaffery JM, Chan DC. 2004. Structural basis of mitochondrial tethering by mitofusin complexes. Science 305:858-62

Kuhlenbaumer G, Young P, Hunermund G, Ringelstein B, Stogbauer F. 2002. Clinical features and molecular genetics of hereditary peripheral neuropathies. F. Neurol. 249:1629-50

Labrousse AM, Zappaterra MD, Rube DA, van der Bliek AM. 1999. C. elegans dynamin-related protein DRP-1 controls severing of the mitochondrial outer membrane. Mol. Cell 4:81526

Lawson VH, Graham BV, Flanigan KM. 2005. Clinical and electrophysiologic features of CMT2A with mutations in the mitofusin 2 gene. Neurology 65:197-204

Lee YJ, Jeong SY, Karbowski M, Smith CL, Youle RJ. 2004. Roles of the mammalian mitochondrial fission and fusion mediators Fis1, Drp1, and Opa1 in apoptosis. Mol. Biol. Cell 15:5001-11

Legesse-Miller A, Massol RH, Kirchhausen T. 2003. Constriction and Dnm1p recruitment are distinct processes in mitochondrial fission. Mol. Biol. Cell 14:1953-63

Legros F, Lombes A, Frachon P, Rojo M. 2002. Mitochondrial fusion in human cells is efficient, requires the inner membrane potential, and is mediated by mitofusins. Mol. Biol. Cell 13:4343-54

Legros F, Malka F, Frachon P, Lombes A, Rojo M. 2004. Organization and dynamics of human mitochondrial DNA. 7. Cell Sci. 117:2653-62

Li Z, Okamoto K, Hayashi Y, Sheng M. 2004. The importance of dendritic mitochondria in the morphogenesis and plasticity of spines and synapses. Cell 119:873-87

Lodi R, Tonon C, Valentino ML, Iotti S, Clementi V, et al. 2004. Deficit of in vivo mitochondrial ATP production in OPA1-related dominant optic atrophy. Ann. Neurol. 56:719-23

Malka F, Guillery O, Cifuentes-Diaz C, Guillou E, Belenguer P, et al. 2005. Separate fusion of outer and inner mitochondrial membranes. EMBO Rep. 6:853-59

Margineantu DH, Gregory Cox W, Sundell L, Sherwood SW, Beechem JM, Capaldi RA. 2002. Cell cycle dependent morphology changes and associated mitochondrial DNA redistribution in mitochondria of human cell lines. Mitochondrion 1:425-35

Mattenberger Y, James DI, Martinou JC. 2003. Fusion of mitochondria in mammalian cells is dependent on the mitochondrial inner membrane potential and independent of microtubules or actin. FEBS Lett. 538:53-59

McMahon HT, Gallop JL. 2005. Membrane curvature and mechanisms of dynamic cell membrane remodelling. Nature 438:590-96

McQuibban GA, Saurya S, Freeman M. 2003. Mitochondrial membrane remodelling regulated by a conserved rhomboid protease. Nature 423:537-41

Meeusen S, McCaffery JM, Nunnari J. 2004. Mitochondrial fusion intermediates revealed in vitro. Science 305:1747-52 
Meeusen SL, Nunnari J. 2005. How mitochondria fuse. Curr. Opin. Cell Biol. 17:389-94

Mozdy AD, McCaffery JM, Shaw JM. 2000. Dnm1p GTPase-mediated mitochondrial fission is a multi-step process requiring the novel integral membrane component Fis1p. 7. Cell Biol. 151:367-80

Nakada K, Inoue K, Ono T, Isobe K, Ogura A, et al. 2001. Inter-mitochondrial complementation: mitochondria-specific system preventing mice from expression of disease phenotypes by mutant mtDNA. Nat. Med. 7:934-40

Niemann A, Ruegg M, La Padula V, Schenone A, Suter U. 2005. Ganglioside-induced differentiation associated protein 1 is a regulator of the mitochondrial network: new implications for Charcot-Marie-Tooth disease. F. Cell Biol. 170:1067-78

Nishida K, Takahara M, Miyagishima SY, Kuroiwa H, Matsuzaki M, Kuroiwa T. 2003. Dynamic recruitment of dynamin for final mitochondrial severance in a primitive red alga. Proc. Natl. Acad. Sci. USA 100:2146-51

Nunnari J, Marshall WF, Straight A, Murray A, Sedat JW, Walter P. 1997. Mitochondrial transmission during mating in Saccharomyces cerevisiae is determined by mitochondrial fusion and fission and the intramitochondrial segregation of mitochondrial DNA. Mol. Biol. Cell 8:1233-42

Okamoto K, Shaw JM. 2005. Mitochondrial morphology and dynamics in yeast and multicellular eukaryotes. Annu. Rev. Genet. 39:503-36

Olichon A, Baricault L, Gas N, Guillou E, Valette A, et al. 2003. Loss of OPA1 perturbates the mitochondrial inner membrane structure and integrity, leading to cytochrome c release and apoptosis. F. Biol. Chem. 278:7743-46

Olichon A, Emorine LJ, Descoins E, Pelloquin L, Brichese L, et al. 2002. The human dynaminrelated protein OPA1 is anchored to the mitochondrial inner membrane facing the intermembrane space. FEBS Lett. 523:171-76

Ono T, Isobe K, Nakada K, Hayashi JI. 2001. Human cells are protected from mitochondrial dysfunction by complementation of DNA products in fused mitochondria. Nat. Genet. 28:272-75

Osteryoung KW, Nunnari J. 2003. The division of endosymbiotic organelles. Science 302:1698704

Payne M, Yang Z, Katz BJ, Warner JE, Weight CJ, et al. 2004. Dominant optic atrophy, sensorineural hearing loss, ptosis, and ophthalmoplegia: a syndrome caused by a missense mutation in OPA1. Am. F. Ophthalmol. 138:749-55

Pesch UE, Fries JE, Bette S, Kalbacher H, Wissinger B, et al. 2004. OPA1, the disease gene for autosomal dominant optic atrophy, is specifically expressed in ganglion cells and intrinsic neurons of the retina. Invest. Ophthalmol. Vis. Sci. 45:4217-25

Praefcke GJ, McMahon HT. 2004. The dynamin superfamily: universal membrane tubulation and fission molecules? Nat. Rev. Mol. Cell Biol. 5:133-47

Rapaport D, Brunner M, Neupert W, Westermann B. 1998. Fzo1p is a mitochondrial outer membrane protein essential for the biogenesis of functional mitochondria in Saccharomyces cerevisiae. F. Biol. Chem. 273:20150-55

Rojo M, Legros F, Chateau D, Lombes A. 2002. Membrane topology and mitochondrial targeting of mitofusins, ubiquitous mammalian homologs of the transmembrane GTPase Fzo. 7. Cell Sci. 115:1663-74

Santel A, Fuller MT. 2001. Control of mitochondrial morphology by a human mitofusin. 7. Cell Sci. 114:867-74

Satoh M, Hamamoto T, Seo N, Kagawa Y, Endo H. 2003. Differential sublocalization of the dynamin-related protein OPA1 isoforms in mitochondria. Biochem. Biophys. Res. Commun. 300:482-93 
Sesaki H, Jensen RE. 1999. Division versus fusion: Dnm1p and Fzo1p antagonistically regulate mitochondrial shape. 7. Cell Biol. 147:699-706

Sesaki H, Jensen RE. 2001. UGO1 encodes an outer membrane protein required for mitochondrial fusion. 7. Cell Biol. 152:1123-34

Sesaki H, Southard SM, Hobbs AE, Jensen RE. 2003a. Cells lacking Pcp1p/Ugo2p, a rhomboid-like protease required for Mgm1p processing, lose mtDNA and mitochondrial structure in a Dnm1p-dependent manner, but remain competent for mitochondrial fusion. Biochem. Biophys. Res. Commun. 308:276-83

Sesaki H, Southard SM, Yaffe MP, Jensen RE. 2003b. Mgm1p, a dynamin-related GTPase, is essential for fusion of the mitochondrial outer membrane. Mol. Biol. Cell 14:2342-56

Shaw JM, Nunnari J. 2002. Mitochondrial dynamics and division in budding yeast. Trends Cell Biol. 12:178-84

Smirnova E, Griparic L, Shurland DL, van Der Bliek AM. 2001. Dynamin-related protein drp1 is required for mitochondrial division in mammalian cells. Mol. Biol. Cell 12:2245-56

Sugioka R, Shimizu S, Tsujimoto Y. 2004. Fzo1, a protein involved in mitochondrial fusion, inhibits apoptosis. 7. Biol. Chem. 279:52726-34

Suzuki M, Jeong SY, Karbowski M, Youle RJ, Tjandra N. 2003. The solution structure of human mitochondria fission protein Fis1 reveals a novel TPR-like helix bundle. F. Mol. Biol. 334:445-58

Suzuki M, Neutzner A, Tjandra N, Youle RJ. 2005. Novel structure of the $\mathrm{N}$ terminus in yeast Fis1 correlates with a specialized function in mitochondrial fission. F. Biol. Chem. 280:21444-52

Szabadkai G, Simoni AM, Chami M, Wieckowski MR, Youle RJ, Rizzuto R. 2004. Drp-1dependent division of the mitochondrial network blocks intraorganellar $\mathrm{Ca}^{2+}$ waves and protects against $\mathrm{Ca}^{2+}$-mediated apoptosis. Mol. Cell 16:59-68

Tieu Q, Nunnari J. 2000. Mdv1p is a WD repeat protein that interacts with the dynamin-related GTPase, Dnm1p, to trigger mitochondrial division. F. Cell Biol. 151:353-66

Tieu Q, Okreglak V, Naylor K, Nunnari J. 2002. The WD repeat protein, Mdv1p, functions as a molecular adaptor by interacting with Dnm1p and Fis1p during mitochondrial fission. 7. Cell Biol. 158:445-52

Tondera D, Czauderna F, Paulick K, Schwarzer R, Kaufmann J, Santel A. 2005. The mitochondrial protein MTP18 contributes to mitochondrial fission in mammalian cells. 7 . Cell Sci. 118:3049-59

A study of the role of mitochondria in the neuromuscular junction.
Verstreken P, Ly CV, Venken KJ, Koh TW, Zhou Y, Bellen HJ. 2005. Synaptic mitochondria are critical for mobilization of reserve pool vesicles at Drosophila neuromuscular junctions. Neuron 47:365-78

Wong ED, Wagner JA, Gorsich SW, McCaffery JM, Shaw JM, Nunnari J. 2000. The dynaminrelated GTPase, $\mathrm{Mgm} 1 \mathrm{p}$, is an intermembrane space protein required for maintenance of fusion competent mitochondria. F. Cell Biol. 151:341-52

Wong ED, Wagner JA, Scott SV, Okreglak V, Holewinske TJ, et al. 2003. The intramitochondrial dynamin-related GTPase, Mgm1p, is a component of a protein complex that mediates mitochondrial fusion. 7. Cell Biol. 160:303-11

Yoon Y, Krueger EW, Oswald BJ, McNiven MA. 2003. The mitochondrial protein hFis1 regulates mitochondrial fission in mammalian cells through an interaction with the dynaminlike protein DLP1. Mol. Cell Biol. 23:5409-20

Yoon Y, Pitts KR, McNiven MA. 2001. Mammalian dynamin-like protein DLP1 tubulates membranes. Mol. Biol. Cell 12:2894-905 
Zuchner S, De Jonghe P, Jordanova A, Claeys KG, Guergueltcheva V, et al. 2006. Axonal neuropathy with optic atrophy is caused by mutations in mitofusin 2. Ann. Neurol. 59:27681

Zuchner S, Mersiyanova IV, Muglia M, Bissar-Tadmouri N, Rochelle J, et al. 2004. Mutations in the mitochondrial GTPase mitofusin 2 cause Charcot-Marie-Tooth neuropathy type 2A. Nat. Genet. 36:449-51

Zuchner S, Vance JM. 2005. Emerging pathways for hereditary axonopathies. 7. Mol. Med.

The identification of $\mathrm{Mfn} 2$ mutations as a cause of CMT2A. $83: 935-43$ 
R

Annual Review of

Cell and

Contents

Developmental

Biology

Volume 22, 2006

From Nuclear Transfer to Nuclear Reprogramming: The Reversal of Cell Differentiation 7.B. Gurdon ..................................................................

How Does Voltage Open an Ion Channel?

Francesco Tombola, Medha M. Pathak, and Ehud Y. Isacoff .....

Cellulose Synthesis in Higher Plants

Chris Somerville

Mitochondrial Fusion and Fission in Mammals

David C. Chan

Agrobacterium tumefaciens and Plant Cell Interactions and Activities

Required for Interkingdom Macromolecular Transfer

Colleen A. McCullen and Andrew N. Binns

Cholesterol Sensing, Trafficking, and Esterification

Ta-Yuan Chang, Catherine C.Y. Chang, Nobutaka Obgami, and Yosbio Yamauchi

Modification of Proteins by Ubiquitin and Ubiquitin-Like Proteins Oliver Kerscher, Rachael Felberbaum, and Mark Hochstrasser ....

Endocytosis, Endosome Trafficking, and the Regulation of Drosopbila

Development

Janice A. Fischer, Suk Ho Eun, and Benjamin T. Doolan

Tight Junctions and Cell Polarity

Kunyoo Shin, Vanessa C. Fogg, and Ben Margolis

In Vivo Migration: A Germ Cell Perspective

Prabhat S. Kunwar, Daria E. Siekbaus, and Ruth Lehmann

Neural Crest Stem and Progenitor Cells

Jennifer F. Crane and Paul A. Trainor 
Of Extracellular Matrix, Scaffolds, and Signaling: Tissue Architecture

Regulates Development, Homeostasis, and Cancer

Celeste M. Nelson and Mina 7. Bissell

Intrinsic Regulators of Pancreatic $\beta$-Cell Proliferation

Feremy 7. Heit, Satyajit K. Karnik, and Seung K. Kim

Epidermal Stem Cells of the Skin

Cédric Blanpain and Elaine Fuchs...

The Molecular Diversity of Glycosaminoglycans Shapes Animal

Development

Hannes E. Bülow and Oliver Hobert

Recognition and Signaling by Toll-Like Receptors

A. Phillip West, Anna Alicia Koblansky, and Sankar Ghosh

The Formation of TGN-to-Plasma-Membrane Transport Carriers

Frédéric Bard and Vivek Malhotra

Iron-Sulfur Protein Biogenesis in Eukaryotes: Components and

Mechanisms

Roland Lill and Ulrich Müblenhoff ....

Intracellular Signaling by the Unfolded Protein Response

Sebastián Bernales, Feroz R. Papa, and Peter Walter

The Cellular Basis of Kidney Development

Gregory R. Dressler

Telomeres: Cancer to Human Aging

Sheila A. Stewart and Robert A. Weinberg

The Interferon-Inducible GTPases

Sascha Martens and Fonathan Howard

What Mouse Mutants Teach Us About Extracellular Matrix Function

A. Aszódi, Kyle R. Legate, I. Nakchbandi, and R. Fässler

Caspase-Dependent Cell Death in Drosophila

Bruce A. Hay and Ming Guo

Regulation of Commissural Axon Pathfinding by Slit and its Robo

Receptors

Barry 7. Dickson and Giorgio F. Gilestro

Blood Cells and Blood Cell Development in the Animal Kingdom

Volker Hartenstein

Axonal Wiring in the Mouse Olfactory System

Peter Mombaerts 